\title{
Impressions of HIV risk online: Brain potentials while viewing online dating profiles
}

\author{
Ralf Schmälzle ${ }^{1,2} \cdot$ Martin A. Imhof ${ }^{2} \cdot$ Alex Kenter $^{2} \cdot$ Britta Renner $^{2} \cdot$ Harald T. Schupp ${ }^{2}$
}

Published online: 10 June 2019

(C) The Psychonomic Society, Inc. 2019

\begin{abstract}
There is an increasing trend to use online dating to meet potential partners. Previous studies in off-line contexts indicate that people may judge the risk of sexually transmitted infections based on a person's appearance. Online dating profiles commonly present profile pictures and verbal self-descriptions. To examine the integration of verbal and visual risk information, the current event-related potential (ERP) study used a simulated dating platform in which verbal-descriptive information (low vs. high verbal risk) was presented, followed by a photograph (low vs. high visual risk). Results indicated main effects of verbal and visual risk. Specifically, high-risk compared with low-risk verbal profiles elicited a relative negative shift over occipitoparietal sensor sites between $260 \mathrm{~ms}$ and $408 \mathrm{~ms}$. Furthermore, a sustained occipital negativity $(132-500 \mathrm{~ms})$ and central positivity $(156-272 \mathrm{~ms}) \mathrm{was}$ observed for high as compared with low visual risk profiles. There was also evidence for the integration of verbal and visual risk formation, as indicated by distinct positive ERP shift occurred between $272 \mathrm{~ms}$ and $428 \mathrm{~ms}$ over anterior temporal regions when a high-risk photograph was preceded by high-risk verbal information. This suggests that verbal-descriptive information is integrated with visual appearance early in the processing stream. The distinct response for high verbal and visual information extends the notion of an alarm function ascribed to risk perception by demonstrating integration about multiple sources. Simulating online dating platforms provides a useful tool to examine intuitive risk perception.
\end{abstract}

Keywords Online dating $\cdot$ Risk perception $\cdot \mathrm{HIV} \cdot \mathrm{EEG} \cdot$ Intuition $\cdot$ Affect

\section{Introduction}

Many dates, sexual contacts, and relationships are initiated via dating websites (Rosenfeld \& Thomas, 2012). Users of such websites actively browse through profiles and make consequential choices about whom to contact and potentially meet in real life (Bruch, Feinberg, \& Lee, 2016; Finkel, Eastwick, Karney, Reis, \& Sprecher, 2012; Heino, Ellison, \& Gibbs, 2010; Lin \& Lundquist, 2013). Although online dating is highly efficient for finding a partner, real and perceived risks need to be addressed. For instance, users perceive online dating as being risky in terms of contracting sexually transmitted infections (STIs) and for meeting untrustworthy individuals

Ralf Schmälzle

ralf.schmaelzle@gmail.com

1 Department of Communication, College of Arts and Sciences, Michigan State University, East Lansing, MI, USA

2 Department of Psychology, University of Konstanz, Konstanz, Germany
(Couch \& Liamputtong, 2007; Couch, Liamputtong, \& Pitts, 2012; Siegel, Lekas, Onaga, Verni, \& Gunn, 2017). The perceived risk of dating and seeking sex partners online seems to be grounded in reality as several studies reported that using online dating services is associated with sexual risk behavior (Cabecinha et al., 2017; Buhi et al., 2013; McFarlane, Bull, \& Rietmeijer, 2000; Ortiz-Martínez, Buelvas-Pérez, MartínezTorres, Vásquez-Rada, \& Carrascal-Angelo, 2018). Consequently, public health experts warn that the steady rise of online dating may facilitate the spread of STIs (Clark, 2015; Greenwood \& Agarwal, 2016; Heijman et al., 2016; McFarlane et al., 2000). With regard to HIV infections, recent numbers still show only marginal declines in Western countries and increases in Eastern Europe and central Asia (UNAIDS, 2016). The current study examines the neural correlates of how impressions of risk are formed online, focusing on the integration of information provided in verbal descriptions and visual profile pictures.

Previous research on mate choice and sexual decisionmaking in off-line contexts suggests that people may rely on intuition to gauge "who is risky and who is safe." Qualitative studies, survey research, and interviews with people who 
contracted HIV through unprotected sex point to a number of illusory control strategies that may nurture a false sense of control, as, for example, quick screenings of a partner's personality or past sexual behavior (Gold, Karmiloff-Smith, Skinner, \& Morton, 1992; Keller, 1993; Klepinger, Billy, Tanfer, \& Grady, 1993; Maticka-Tyndale, 1991; Prestage et al., 2016). Studies of the sociocognitive factors underlying these phenomena (Agocha \& Cooper, 1999; Eleftheriou, Bullock, Graham, Stone, \& Ingham, 2016; Fishbein, Hennessy, Yzer, \& Curtis, 2004; Hennessy, Fishbein, Curtis, $\&$ Barrett, 2007) have recently been complemented by research examining the neural processes of how people evaluate HIV risk during "first sight" (Renner, Gamp, Schmälzle, \& Schupp, 2015; Schmälzle, Renner, \& Schupp, 2017). To shed light on implicit processes that unfold while people form impressions, functional imaging and event-related brain potentials (ERPs) have been recorded while participants were exposed to photographs of unacquainted persons. With regard to speed of processing, the data from ERP studies showed that differences between individuals subsequently judged as risky or safe emerge early in the processing stream $(<300 \mathrm{~ms})$. In addition, portraits of individuals perceived as risky as compared with safe elicited significantly larger late positive potentials (LPPs), a cortical marker of affective evaluation (Schupp, Flaisch, Stockburger, \& Junghöfer, 2006; Renner, Schmälzle, \& Schupp, 2012; Schmälzle, Schupp, Barth, \& Renner, 2011). Furthermore, the hypothesis that these risk perceptions emerge spontaneously has been corroborated by studies in which participants performed a memory task unrelated to HIV, yet electrophysiological and hemodynamic responses still showed a relationship to HIV risk, which was assessed in a subsequent explicit condition (Häcker, Schmälzle, Renner, \& Schupp, 2014; Schmälzle et al., 2011). In sum, increasing evidence suggests that intuitive impressions influence HIV risk perception, which may have severe real-world consequences, reminiscent of the role first impressions play in professional, educational, and legal contexts (Bellew \& Todorov, 2007; Brooks, Huang, Kearney, \& Murray, 2014; Eberhardt, Davies, Purdie-Vaughns, \& Johnson, 2006; Olson \& Marshuetz, 2005; Todorov, 2017; Uleman, Blader, \& Todorov, 2006a, 2006b).

Work on how mate choice unfolds online has examined how users form impressions based on the information available in a potential partner's profile. Past research has focused predominantly on how people represent or misrepresent themselves online, how users spot deception, and which filter strategies are used during profile browsing, which has been compared to "shopping" (Bruch et al., 2016; Heino et al., 2010; Toma, Hancock, \& Ellison, 2008). However, aside from general perceptions of risk associated with online dating, little is known about the underlying processes that lead to the perception of risk or safety (i.e., when people browse partner profiles). Online dating profiles typically consist of verbal information, such as demographic data and brief self-descriptions, and a visual profile picture. People must make sense of this sparse information to assess a potential partner's risk by recruiting knowledge stored in person and social world memory systems (Adams, Ambady, Nakayama, \& Shimojo, 2010; Gilbert, 1998; Linville \& Carlston, 1994). Research in social, cognitive, and affective neuroscience has amassed a wealth of knowledge about the mechanisms of impressions based on visual appearance (Adolphs, 2003, 2009; Allison, Puce, Spencer, \& McCarthy, 1999; Ambady \& Rosenthal, 1992; Naumann, Vazire, Rentfrow, \& Gosling, 2009; Todorov, 2017), and recent work has begun to specifically examine how verbal biographical and visual information are integrated in associative person memory (Calder, Rhodes, Johnson, \& Haxby, 2011; Collins, Koski, \& Olson, 2016; Collins \& Olson, 2014a, 2014b). Event-related potential studies demonstrated that face processing was changed when the faces were associated in a preceding learning phase with affective as compared with neutral autobiographical information (Abdel Rahman, 2011; Baum, Rabovsky, Rose, \& Abdel Rahman, 2018; Luo, Wang, Dzhelyova, Huang, \& Mo, 2016; Suess, Rabovsky, \& Rahman, 2015). Functional imaging studies of multiple sources of personal information point to increased activations in the anterior temporal lobe (Olson, McCoy, Klobusicky, \& Ross, 2013), and intracranial recordings revealed sensitive responding to faces across multiple recording sites of the ventral stream between $100 \mathrm{~ms}$ and $600 \mathrm{~ms}$ after stimulus onset (Barbeau et al., 2008; Tsuchiya, Kawasaki, Oya, Howard III, \& Adolphs, 2008). However, the nexus between social cognition and health-risk perception has rarely been studied, particularly not in the context of risk perception online.

The present study examines HIV risk perception in the context of online dating, focusing on the integration of verbal-descriptive information and visual appearance of the profile picture. Thus, we created a dating platform that resembled online dating services and presented profiles that systematically varied verbal and visual information related to HIV risk (see Fig. 1). Specifically, high-risk profiles described individuals with many sexual partners and statements indicative of carelessness and irresponsibility, which previous work identified as key elements of a high-risk stereotype (Renner \& Schwarzer, 2003). This information was credibly embedded under a rubric labeled "pillow talk" and was presented alongside other relevant and irrelevant cues such as nicknames, age, location, and hobbies. The low-risk profiles, on the other hand, described individuals with cautious traits and safe past behaviors. Each dating profile also contained a picture of the person, which always succeeded the verbal information. Similar to verbal risk information, the photographs were selected to form categories of low and high HIV risk based on visual appearance, which was evaluated in 


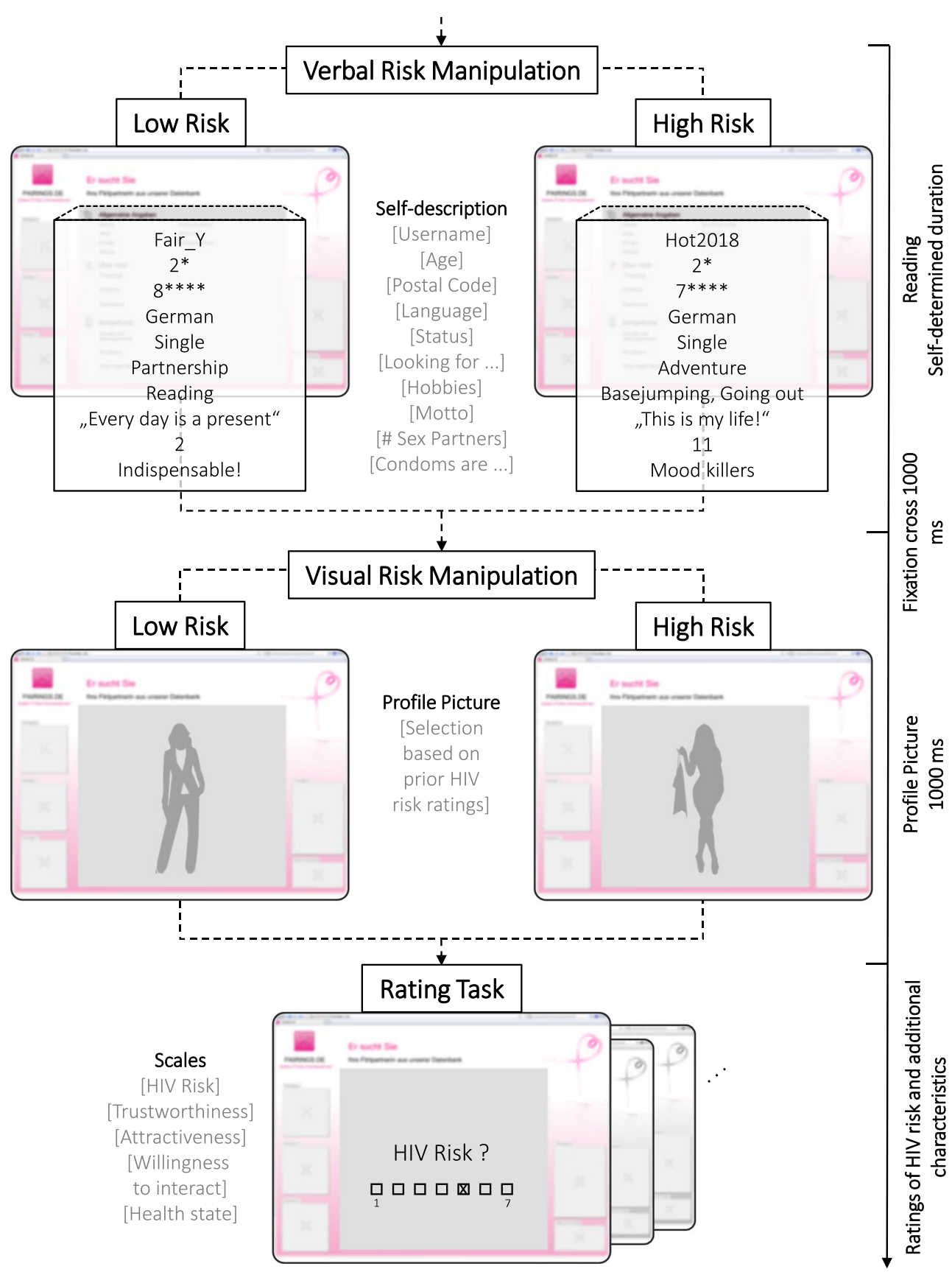

Fig. 1 Schematic illustration of the experimental design and timeline of an experimental trial. Event-related brain potentials are derived relative to the onset of the profile picture. Presentation software was used to manipulate and display the verbal and visual risk information within a carefully designed website. The website included typical user interface features, such as a browser window with address bar, design features (e.g., logos), as well as buttons presumably directing to social media services. To create unique verbal profiles, the text fields were manipulated by assigning values that conveyed a low or high risk feature. Text fields included a username, demographic information, relationship status, hobbies, motto, and a rubric titled "pillow talk," which included information on sexual

previous work (Barth, Schmälzle, Renner, \& Schupp, 2013; Häcker et al., 2014; Renner et al., 2012; Schmälzle et al., 2011). Thus, the experimental design behavior (cf. Renner \& Schwarzer, 2003). The profile pictures were presented after the verbal information and paired with either a low or a high verbal risk profile. Assignment to the low and high visual risk categories was based on previous ratings of HIV risk. For copyright reasons, only schematic images and a redacted website design are shown. After browsing the profile and viewing the images, participants provided ratings of HIV risk as well as additional person characteristics (trustworthiness, attractiveness, willingness to interact, and health state). Two parallel versions of the website were created for male and female participants, respectively

enabled us to study whether and how verbal risk information affects the subsequent neural processing of risk based on visual appearance. 
Previous work suggests that risky-looking pictures elicit brain responses compatible with the hypothesized alarm function of risk perception (i.e., a fast and affective reaction; Renner et al., 2012; Schmälzle, Renner, \& Schupp, 2012, Schmälzle et al., 2017). Furthermore, based on the finding that face processing can be modulated by affective autobiographical information (Suess et al., 2015), one might assume that high verbal risk information affects perceptual processing in particular with regard to an occipital negativity between $200 \mathrm{~ms}$ and $350 \mathrm{~ms}$. However, with respect to the integration of verbal and visual information, multiple types of relationships are possible. For example, if brain responses to the photographs are primarily sensitive to the consistency or inconsistency between the verbal and visual information, then conditions in which a mismatch occurs ("low verbal-high visual risk" and "high verbal-low visual risk") should lead to distinct ERP responses as compared with the matching conditions. Several ERP components have been associated with such violations of expectancy in social cognition research, including P2, N2, and P3/LPP components (Hansen, Steffens, Rakic, \& Wiese, 2017; Osterhout, Bersick, \& McLaughlin, 1997; White, Crites Jr, Taylor, \& Corral, 2009). Alternatively, it may be that a risky-looking photograph prompts particularly strong reactions if it matches or confirms a risky verbal description. Such a finding would be compatible with the alarm function ascribed to risk perception from a functional-theoretic perspective (Schmälzle et al., 2017; Slovic, Finucane, Peters, \& MacGregor, 2004) and demonstrate integration of information from multiple sources early in the processing stream.

\section{Materials and methods}

\section{Participants}

Forty-five healthy volunteers were recruited on the campus of the University of Konstanz. All participants had normal or corrected-to-normal vision. Four participants were excluded from electrophysiological analysis - one because of data acquisition failure, and three because of excessive artifacts. Thus, analysis reported are based on data from 41 participants (22 women, age: $M=22.1$ years, $S D=2.4$ ). Screening questions related to sexual behavior indicated that 19 participants were in a relationship at the time of the study. Furthermore, on average, participants reported to have had sexual intercourse with around four partners $(M=4.3, S D=4.9)$, rare risky sexual behaviors $(M=6.2, S D=1.1$; scale: 1 [always] to 7 [never]) and occasional sexual intercourse without a condom $(M=4.3, S D=2.1$; scale: 1 [never] to 7 [always]). As expected, the sample showed an optimistic bias and estimated their risk as being lower compared with an average peer of the same age and $\operatorname{sex}(M=2.2, S D=1.1$; scale 1 [significantly below average] to 7 to [significantly above average]). Participants received either monetary reward or course credits for participation and provided written consent to the study protocol, which was approved by the Ethics Review Board of the University of Konstanz.

\section{Experimental paradigm}

In order to examine the impact of personal risk information on the viewing of opposite sex photographs, we created an experimental paradigm that emulated an authentic online dating platform. Specifically, Presentation software was used to display a carefully designed and visually appealing website that included typical user interface features, such as a browser window with address bar, logos, social media buttons (e.g., Twitter, Facebook), user statistics, as well as advertisements (see Fig. 1).

Verbal risk information This simulated dating website first displayed a screen providing verbal information about a potential partner (see Fig. 1). Participants controlled the duration of the verbal profile and continued the trial via a mouse click. The verbal profile provided information such as user name, relationship status, hobbies, a motto statement, and relationship wishes. Furthermore, under a rubric titled "pillow talk," information about sexual history (i.e., number of sex partners and number of one-night stands), as well as attitudes towards condom use (e.g. "Never without" or "Condoms are unromantic") was embedded. A total of 160 different risk profiles were created for males and females separately by systematically varying the risk for contracting HIV and sexually transmitted infections. Specifically, informed by previous research on HIV risk (Renner \& Schwarzer, 2003), 80 high-risk profiles described a person with many sexual partners, infrequent condom use, and attributes of carelessness and irresponsibility, while another 80 profiles presented-low risk information (i.e., consistent condom use, no one-night stands, trustful and responsible). The spreadsheet with the unique feature values for each low-risk and high-risk variable was created by the first author and refined using a team of research assistants matching the target audience (available at github.com/ nomcomm/RiskProfiles_CABN). For each participant, unique high-risk and low-risk profiles were created by randomly drawing from the pool of high-risk and low-risk exemplars of each profile variable (i.e., user name, relationship status, hobbies, etc.) of the respective gender.

Visual risk information After a delay of 1 second displaying a fixation cross, the photograph of an opposite-sex person was presented for 1 second. Two sets (male and female) of 160 photographs each were used. These photographs have already been evaluated in terms of HIV risk in previous studies by opposite-sex raters and were retrieved with permission from 
public databases (Barth et al., 2013; Häcker et al., 2014; Renner et al., 2012; Schmälzle et al., 2011; Schmälzle et al., 2019). To closely resemble real dating platforms, the photographs showed portraits varying in attire, socioeconomic status cues, and situational context features. Furthermore, to be representative of the study target population's age and race, only photographs of Caucasians between 18 and 35 years were included. At the time the study was conducted, the database consisted of 240 male and 240 female photographs, along with HIV risk ratings from at least 40 opposite-sex raters per image. From this pool, we selected 80 male and 80 female photographs with highest HIV risk ratings, and 80 male and 80 female images with lowest HIV risk ratings.

Experimental design Verbal and visual risk information were combined to form four experimental conditions consisting of 40 trials each (i.e., 40 low verbal/low visual risk, 40 low verbal/high visual risk, 40 high verbal/low visual risk, and 40 high verbal/high visual risk). The order of trials varied randomly, and each participant received a unique pairing of verbal and visual risk information, controlled by a MATLAB script that generated individual scenarios pairing verbal profiles (high/low verbal risk, individually assigned from the spreadsheet categories) with visual images (high/low visual risk, as defined by ratings of HIV risk from prior studies).

Self-report measures Following the picture display, participants provided ratings of their impression about the potential partner on perceived HIV risk, trustworthiness, attractiveness, willingness to interact, and perceived health on scales ranging from 1 to 7 . The order of rating scales varied randomly across trials.

\section{Procedure}

To increase credibility of the online dating platform, participants were told that the computer would choose randomly among different versions and layouts of online dating services that were to be compared. Specifically, a dynamic intro sequence was created "connecting" to the dating platform. Two practice trials served to familiarize the participants with the profile and photograph presentation as well as the self-report measures. The experiment then displayed the 160 experimental trials (see example in Fig. 1), which lasted about 50 minutes, including two breaks. Presentation software (Neurobehavioral Systems, Inc., Berkeley, CA) was used to present the stimuli and to synchronize EEG acquisition. Stimuli were shown on a 21-in.' computer screen $(1,024 \times$ 768 resolution) located at a distance of approximately $100 \mathrm{~cm}$ from the participants, and the photographs that were embedded into the dating website spanned a visual angle of about $18 \times 13$ degrees. After navigating the dating platform, participants completed questionnaires that assessed sexual history as well as behavior and beliefs concerning HIV. At the end of the study, they were debriefed and informed that the profile information was fictitious.

\section{EEG data acquisition and analysis}

Electrophysiological data were collected from the scalp using a 257-lead HydroCel Geodesic Sensor Net (EGI: Electrical Geodesics, Inc., Eugene, OR). The EEG was recorded continuously with a sampling rate of $250 \mathrm{~Hz}$, with the vertex sensor as reference electrode, and online filtered from $0.1 \mathrm{~Hz}$ to $100 \mathrm{~Hz}$ using Netstation acquisition software and EGI amplifiers. Impedances were kept below $50 \mathrm{k} \Omega$, as recommended for this type of amplifier. Off-line analyses were performed using BESA (BESA GmbH) and EMEGS (Peyk, De Cesarei, \& Junghöfer, 2011) software packages and in-house MATLAB code (The MathWorks, Inc.). Processing steps included low-pass filtering at $40 \mathrm{~Hz}$, artifact detection, bad sensor interpolation, baseline -correction for prestimulus (100 ms) ERP activity, and conversion to an average reference (Junghöfer, Elbert, Tucker, \& Rockstroh, 2000). Trials with blinks were discarded, and eye-movement artifacts were corrected using the adaptive artifact correction approach implemented in BESA (Ille, Berg, \& Scherg, 2002).

Single-sensor waveform analyses were used to determine statistically significant effects for the processing of the pictures as a function of experimental condition. Specifically, the data from each time point and sensor were submitted separately to a repeated-measures ANOVA, including the within factors verbal risk (low verbal, high verbal) and visual risk (low visual, high visual). To account for the multiple comparisons problem, a cluster-based permutation test (Maris \& Oostenveld, 2007) with $N=1,000$ permutations was performed as implemented in the EMEGS package. Sensor clusters were considered meaningful if they reached a singlesensor inclusion threshold of $p<.05$ and a cluster-level threshold of $p<.05$, corrected for multiple comparisons (see Appendix for EGI sensor numbers forming significant clusters). For descriptive reasons, interaction effects were followed up by dependent $t$ tests using Bonferroni-adjusted significance criterion (i.e., $p<.05 / 4$ ).

\section{Results}

\section{Self-report data}

Perceived HIV risk varied as a function of verbal risk information, $F(1,40)=134.7, p<.001$, partial $\eta^{2}=.77$, as well as visual risk information, $F(1,40)=56.5, p<.001$, partial $\eta^{2}=$ .59. As shown in Fig. 2, both high-risk verbal and visual information was associated with increased HIV risk perception compared with low-risk information. The interaction of 

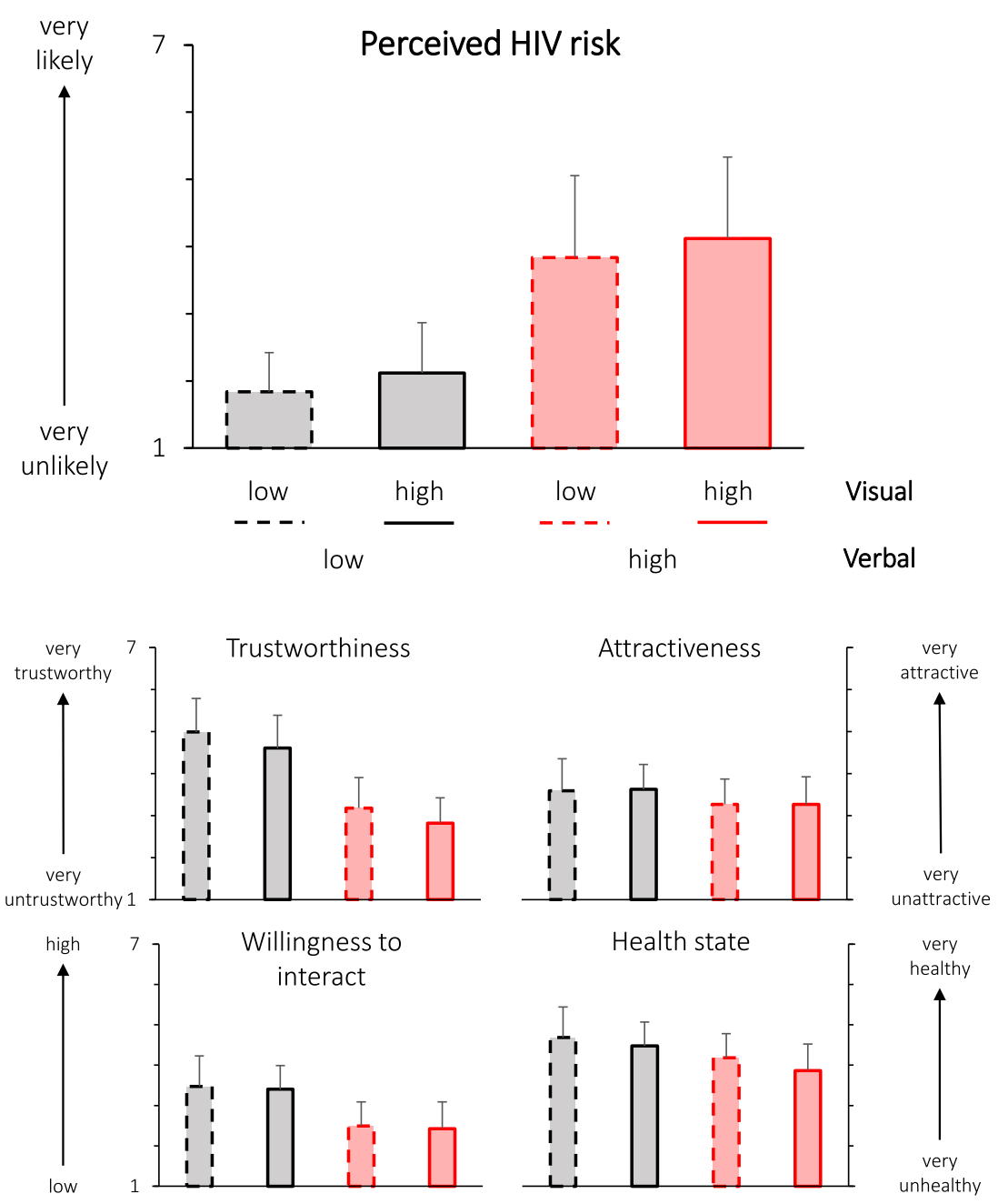

Fig. 2 Top panel: Mean judgments of perceived HIV risk for the combinations of visual and verbal risk categories. Lower panels: Mean judgments of perceived trustworthiness, attractiveness, willingness to interact, and health state. Error bars represent standard deviations

verbal and visual risk information was not significant, $F(1,40)$ $=0.0, n s$.

As expected, a mirroring pattern of results emerged for the analysis of trustworthiness ratings, verbal risk, $F(1,40)=$ $140.2, p<.001$, partial $\eta^{2}=.78$; visual risk, $F(1,40)=68.1$, $p<.001$, partial $\eta^{2}=.63$. For both main effects, high-risk information was perceived as less trustworthy, and vice versa. Attractiveness ratings were modulated by verbal risk information, $F(1,40)=24.5, p<.001$, partial $\eta^{2}=.38$, with low-risk profile individuals being perceived as more attractive. Neither the main effect of visual risk, $F(1,40)=0.9, n s$, nor the interaction of verbal and visual risk was significant, $F(1,40)$ $=0.01, n s$. Similarly, willingness to interact was significantly larger for individuals associated with a low-risk as compared with a high-risk verbal profile: verbal risk, $F(1,40)=62.6$, partial $\eta^{2}=.61$. Neither the main effect visual risk, $F(1,40)=$ $0.04, n s$, nor the interaction of verbal and visual risk was significant, $F(1,40)=0.5$, ns. Perceived health was higher for both low verbal, $F(1,40)=36.9, p<.001$, partial $\eta^{2}=$ .48 , as well as low visual risk information, $F(1,40)=42.2, p<$
.001 , partial $\eta^{2}=.51$, with both factors showing an interaction, $F(1,40)=5.6, p=.02$, partial $\eta^{2}=.12$.

\section{Event-related potentials}

Single-sensor waveform analysis served to identify effects associated with the processing of verbal and visual risk information. As shown in Figs. 3 and 4, differential processing of high-risk compared with low-risk information was indicated by significant ERP cluster effects for both verbal and visual risk information. Furthermore, a significant interaction of verbal and visual risk information appeared with distinct topography, polarity, and latency. This interaction effect was primarily driven by the condition in which the verbal profiles containing high-risk information were followed by a high-risk photograph (see Fig. 5).

Main effect of "verbal risk" Verbal profiles indicating high as compared with low sexual risk information affected the processing of the upcoming photograph independent from the 
Main effect: Verbal Risk

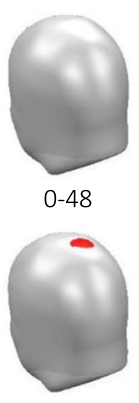

260-308

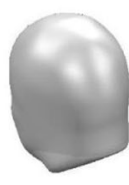

52-100

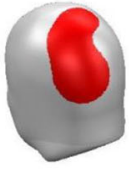

$312-360$

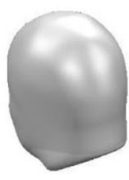

104-152

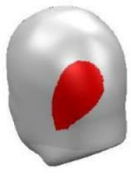

364-412

Time [ms]

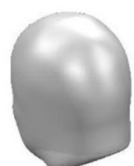

156-204

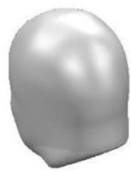

$416-464$

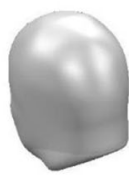

208-256

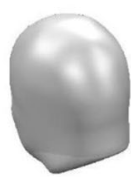

468-516
Cluster

statistic

$p<.05$

corrected

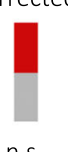

n.s.

\section{ERP waveforms}

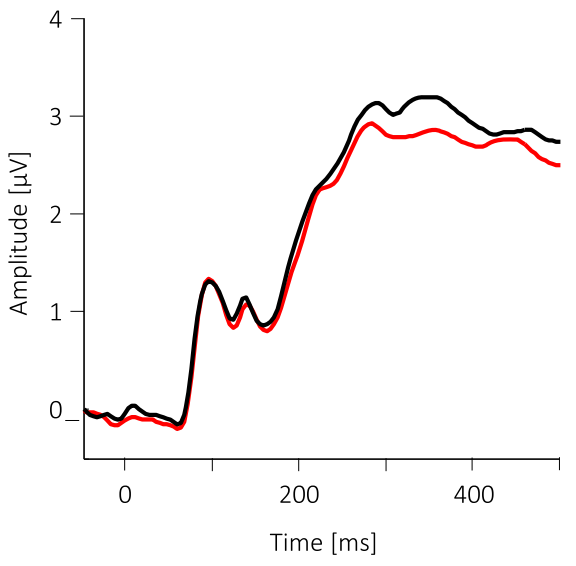

Verbal Risk

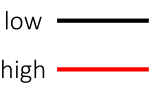

\section{High vs. Low Verbal Risk}

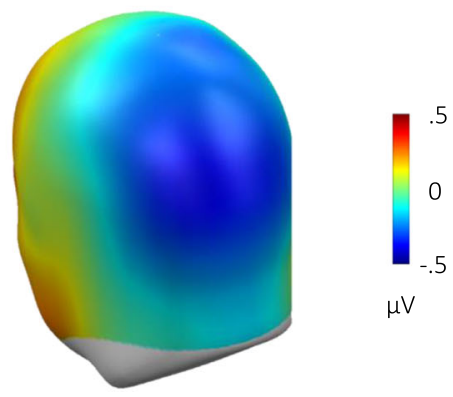

260-408 ms

Fig. 3 Main effect for verbal risk. Top panel: Results of the single sensor waveform analysis. Images show significant cluster effects, thresholded at $p<.05$ for the sensor and cluster level, collapsed across time bins of 48 $\mathrm{ms}$, and shown on a back view tilted $30^{\circ}$ to the left and $30^{\circ}$ backwards for

risk status of the photograph. Specifically, a significant main effect of verbal risk was observed over occipitoparietal sensor sites in a time interval between $260 \mathrm{~ms}$ and $408 \mathrm{~ms}$ (see Fig. 3 ). Inspection of the cluster ERP waveforms and the difference scalp map showed that high verbal risk information is associated with a relative negative shift as compared with low verbal risk information. the occipitoparietal effect. Middle panel: ERP waveforms of the occipitoparietal sensor cluster, separately for low and high verbal risk. Bottom panel: Topographical map of the ERP difference between high and low verbal risk. (Color figure online)
Main effect of "visual risk" Two significant ERP cluster effects were observed comparing the processing of high and low visual risk photographs. As shown in Fig. 4 (left column), the first and stronger cluster effect occurred over right occipital sensor sites between $132 \mathrm{~ms}$ and $500 \mathrm{~ms}$. Similar to the main effect of verbal risk, high-risk information was associated with a relative negative potential as compared with low-risk 


\section{Main effect: Visual Risk}

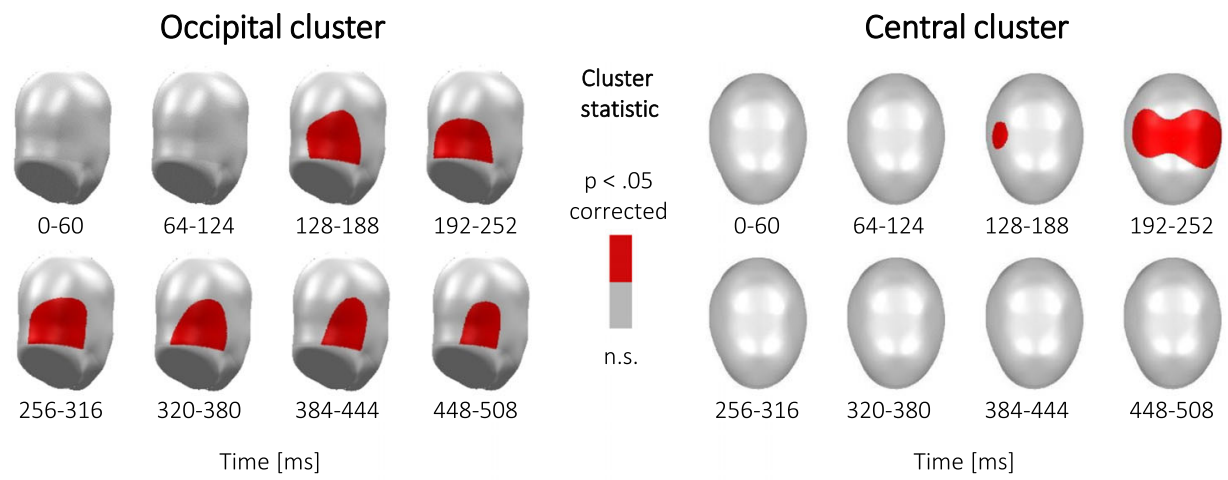

\section{ERP waveforms}
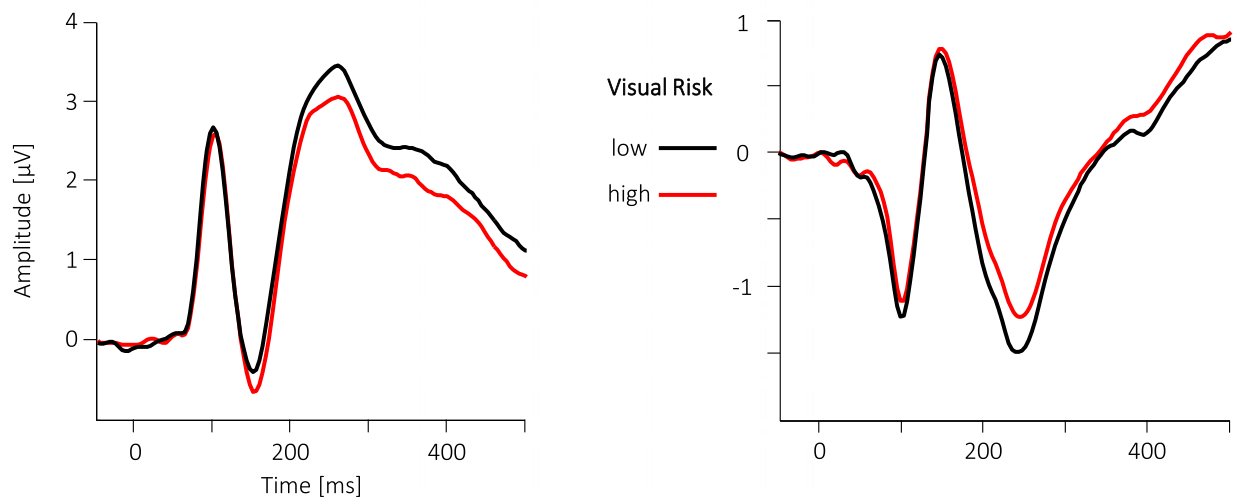

\section{High vs. Low Visual Risk}

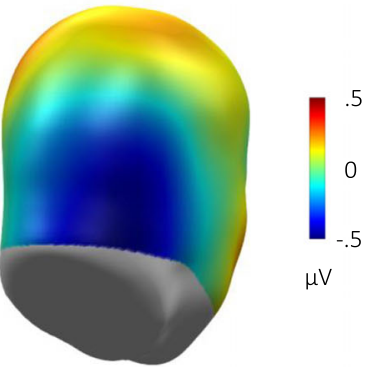

132-500 ms

Fig. 4 Main effect for visual risk. Top panel: Results of the single sensor waveform analysis. Images show significant cluster effects, thresholded at $p<.05$ for the sensor and cluster level, collapsed across time bins of 60 $\mathrm{ms}$, and shown on a back view tilted $30^{\circ}$ to the right for the occipital effect, and a top view for the central effect. Middle panel: ERP waveforms

information. The second main effect of visual risk was observed in a bilateral centrally located cluster between $156 \mathrm{~ms}$ and $272 \mathrm{~ms}$, and the visual risk effect occurred with reversed polarity (i.e., high visual risk was associated with an increased positivity; Fig. 4, right column).

Interaction effects of "verbal and visual risk" A significant interaction of verbal and visual risk was observed in a right ${ }^{1}$

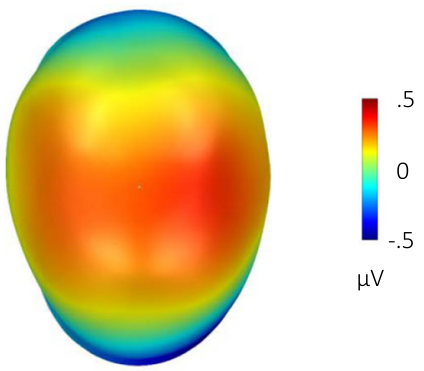

156-272 ms

of the occipital (left) and central (right) sensor cluster, separately for low and high visual risk. Bottom panel: Topographical maps of the ERP difference between high and low visual risk showing the occipital (left) and central (right) effects. (Color figure online)

anterior temporal cluster in a time interval from $272 \mathrm{~ms}$ to $428 \mathrm{~ms}$ (see Fig. 5). For descriptive reasons, the interaction was followed up for both sources of risk (i.e., verbal and visual) separately. Towards this end, mean amplitudes were extracted for this cluster across all sensors and time points contributing to the effect, separately for the four conditions. The processing of high visual risk information elicited a significant relative positive potential shift when preceded by high 
compared with low verbal risk information, $t(40)>-3.24, p=$ .002 . Similarly, when preceded by high verbal risk information, high as compared with low visual risk was associated with a relative positivity, $t(40)>-3.02, p=.004$. The complementary $t$ tests for low-risk conditions were not significant in both cases, $t \mathrm{~s}(40)<1.9, n s$.

Exploratory analysis: Gender differences Post hoc analyses were conducted to explore possible gender differences in processing and evaluating HIV risk. With regard to HIV risk ratings, 2 (verbal risk) $\times 2$ (visual risk) $\times 2$ (gender) ANOVAs revealed no significant interaction involving the factor gender $(F \mathrm{~s}<1.6, p>.2)$. To parallel the self-report analyses with ERPs, mean amplitudes for significant main and interaction effects were extracted across all sensors and time points contributing to the effect. For the main effect of verbal risk, the 2 (verbal risk) $\times 2$ (gender) ANOVA indicated no significant interaction with gender, $F(1,39)=0.05$, $n s$. Similarly, for the main effect of visual risk in the occipital cluster, the 2 (visual risk) $\times 2$ (gender) ANOVA showed no significant interaction effect, $F(1,39)=1.1$, ns. However, there was a significant Visual Risk $\times$ Gender interaction for the centrally located sensor cluster, $F(1,39)=5.1, p=.029$, partial $\eta^{2}=.12$. Although follow-up tests confirmed the effect for both genders, the effect appeared larger in men than in women, $F(1,18)=16.9, p<.001$, and $F(1,21)=4.6, p<$ .05 , respectively. With regard to the interaction effect, a significant three-way interaction of Verbal Risk $\times$ Visual Risk $\times$ Gender was observed, $F(1,39)=4.6, p=.038$, partial $\eta^{2}=.11$. Follow-up analyses showed the effect for women, $F(1,21)=$ $14.8, p=.001$, but not for men, $F(1,18)=0.4$, ns. Together, these exploratory analyses of gender differences suggest largely similar effects with an interesting gender difference in the anterior temporal cluster where verbal and visual risk information interacted. However, given the sample size and experimental design, which was not optimized to reveal gender differences, these findings await replication in future research.

\section{Discussion}

Online dating profiles consist of verbal and visual information, and mate-seeking users must integrate both sources to form a coherent overall impression (Bruch et al., 2016; Finkel et al., 2012). The present study examined how verbaldescriptive information about the riskiness of a person affects

\footnotetext{
${ }^{1}$ While the cluster statistic was not significant over corresponding left hemispheric sensor sites, we conducted an exploratory analysis over the left hemisphere (EGI sensor numbers 241, 244, 245,246, 248, 249, 250, 252, 253, 254; time window $280-400 \mathrm{~ms}$ ), revealing a relative positive shift for the high verbal and visual risk condition compared with the three other conditions, $t \mathrm{~s}(40)>-2.0, p<.05$
}

spontaneous processing of a person's visual appearance as measured by event-related brain potentials. Three main findings emerged: First, the processing of the photograph was affected by the preceding verbal risk information independent from the risk status of the photograph. Specifically, high-risk verbal profiles elicited a relative negative shift over occipitoparietal sensor sites between $260 \mathrm{~ms}$ and $408 \mathrm{~ms}$. Second, photographs from the high as compared with the low visual risk category were associated with a sustained negative shift between $132 \mathrm{~ms}$ and $500 \mathrm{~ms}$ over occipital sensor regions and a positive shift over central sensor sites between $156 \mathrm{~ms}$ and $272 \mathrm{~ms}$, similarly pronounced for low and high verbal risk conditions. Third, after reading the profile of a person matching a high-risk HIV stereotype, viewing a photograph from the high-risk category prompted an early $(\sim 270$ ms) anterior-temporal positive ERP shift compared with the three other conditions. This work extends previous research on intuitive risk perception to a potentially consequential realworld setting and establishes a platform to examine health risk perception and social impression formation in a use-inspired basic science approach (Stokes, 2011).

A main finding of the present study is that verbal risk information affects the processing of the upcoming photograph. This finding resonates with previous ERP research examining the impact of affective personality information on face processing (Abdel Rahman, 2011; Baum et al., 2018; Luo et al., 2016; Suess et al., 2015). This line of research relies on a learning phase in which negative, neutral, and positive autobiographical information is repeatedly paired with faces. Notably, in the testing phase, ERPs were collected to face processing in a variety of task conditions, such as, for instance, a gender task that did not require participants to recollect the autobiographical information. The findings indicate that faces associated with negative and positive compared with neutral autobiographical information elicited a larger relative negativity over posterior sensor regions between $\sim 200 \mathrm{~ms}$ and $400 \mathrm{~ms}$ (Abdel Rahman, 2011; Luo et al., 2016; Suess et al., 2015). Acknowledging differences in experimental methodology, the similarity in findings is striking in that high verbal risk profiles elicit an ERP modulation similar in polarity, topography, and latency to emotional autobiographical information. Overall, providing information about sexual risk behavior, the present findings add further evidence to the notion that autobiographical knowledge about a person affects the processing of the visual appearance of the person.

Prior work using only photographs found that viewing high-risk photographs prompted a relatively negative deflection over occipital sites (Renner et al., 2012; Schmälzle et al., 2011), and the relative occipital negativity towards riskylooking profile pictures corroborates this effect. However, within the current work, negativity emerged earlier $(\sim 132$ $\mathrm{ms}$ ) and lasted longer ( $\sim 00 \mathrm{~ms})$. Furthermore, it seems possible that the relative shift associated with high-risk 


\section{Interaction: Verbal x Visual Risk}

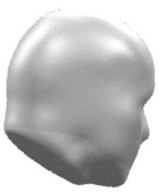

$0-48$

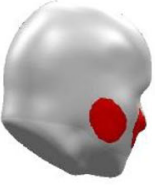

260-308

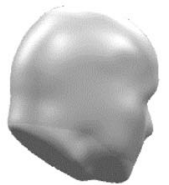

$52-100$

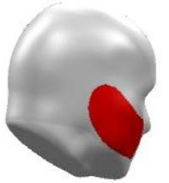

$312-360$

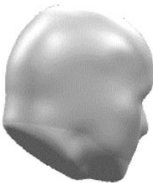

104-152

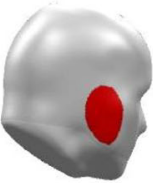

364-412

Time $[\mathrm{ms}]$

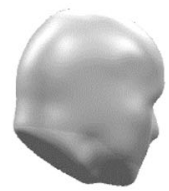

156-204

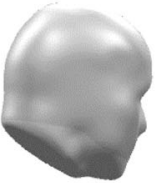

416-464

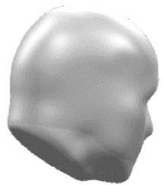

208-256

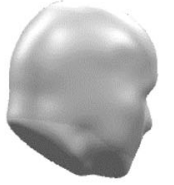

468-516
Cluster

statistic

$p<.05$

corrected

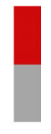

n.s.

\section{ERP waveforms}

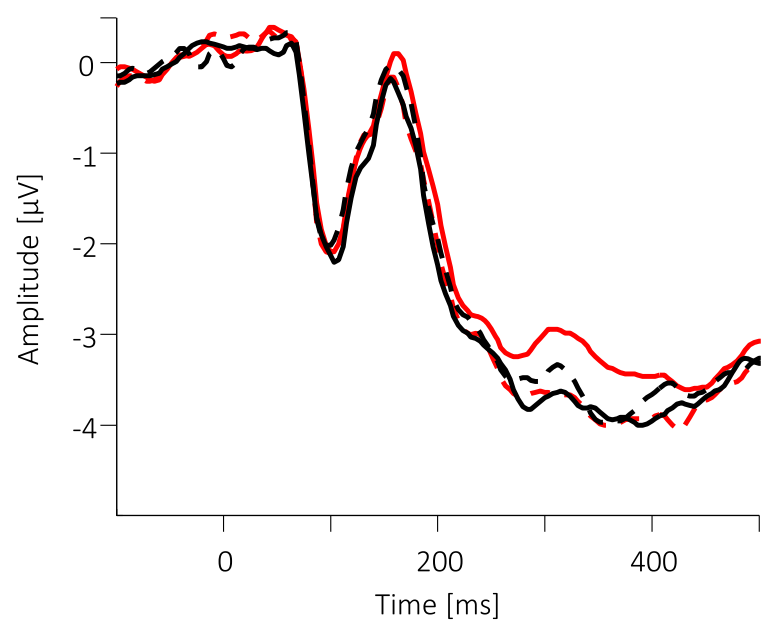

Risk Information

Verbal Visual

low - - - low

low high

high - - low

high $\longrightarrow$ high

\section{High risk vs. other conditions}

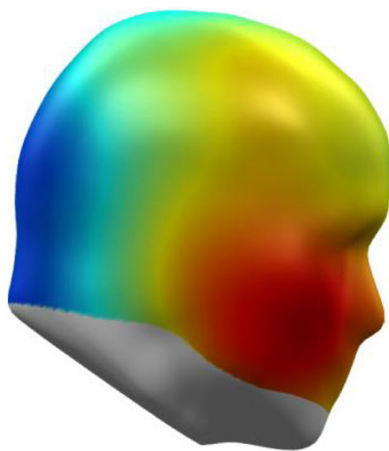

$272-428 \mathrm{~ms}$

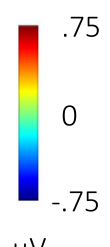

$\mu \mathrm{V}$
Fig. 5 Verbal $\times$ Visual Risk interaction effect. Top panel: Results of the single sensor waveform analysis. Images show significant cluster effects, thresholded at $p<.05$ for the sensor and cluster level, collapsed across time bins of $48 \mathrm{~ms}$, and shown on a right view tilted $20^{\circ}$ to the left for the anterior temporal effect. Middle panel: ERP waveforms of the anterior temporal sensor cluster, separately for the four conditions. Bottom panel: Topographical map of the ERP difference between high visual/high verbal risk and the three other conditions. (Color figure online) 
photographs over central sensor regions reflects polarity reversal, a hypothesis awaiting future research. Methodological differences between the current and previous work may explain differences in the appearance of early modulation effects. Previous research dealt with visual appearance only and did not include verbal risk information. Task set and instruction can have profound effects on speed of processing. For instance, research on the processing of natural scenes revealed that the recognition of a single picture as compared with a higher order semantic classification task speeded the onset latency of ERPs by 30-40 ms (Delorme, Rousselet, Macé, \& Fabre-Thorpe, 2004). One may speculate that methodological differences, in particular the activation of riskrelated knowledge structures by the verbal profile (cf. Collins \& Olson, 2014a, 2014b), account for the observed differences in the latency of the occipital negativity elicited by risky-looking persons. This reasoning could also account for another difference between the current findings and previous work: Previous studies found that LPP amplitudes increased with higher ratings of HIV risk (Renner et al., 2012; Schmälzle et al., 2012; Schmälzle et al., 2011), whereas no such effect was seen in the current data. In sum, the main effect of visual risk provides further evidence for the notion that HIV risk impressions appear to be embedded in a stereotypical set of beliefs about negative personality attributes (Renner \& Schwarzer, 2003), which are activated during first sight.

Beyond the main effects of verbal and visual risk, a novel finding is revealed by the significant interaction of both factors, reflected by an ERP difference over anterior-temporal sites between $272 \mathrm{~ms}$ and $428 \mathrm{~ms}$. Critically, this effect was specific to the condition conveying high verbal and high visual risk - that is, the maximum risk information possible. Although the effect reached significance over the right hemisphere, a similar pattern of ERP modulation was seen over corresponding left hemispheric sites at the same latency in exploratory analysis. Evidence from anatomical, lesion, ani$\mathrm{mal}$, and functional imaging studies points to the anterior temporal lobe as critical for social cognition and person memory (Olson et al., 2013), and as a "switchboard for conceptual knowledge" (Lambon Ralph, Jefferies, Patterson, \& Rogers, 2016; Wang et al., 2017). Specifically, functional imaging studies revealed activation of the anterior temporal regions in conditions in which multiple pieces of information had to be integrated, and facial identity was linked to semantic, biographic, and emotional knowledge (Olson et al., 2013; Wang et al., 2017). Furthermore, intracranial recordings in epileptic patients indicate ERPs over anterior temporal regions to faces, peaking around $350 \mathrm{~ms}$ after stimulus onset, with larger amplitudes to famous compared with unknown faces (Barbeau et al., 2008; Trautner et al., 2004). Viewed from this perspective, reading a dating profile with name, hobbies, and sexual behavior may create a transient person representation, which is then integrated with the incoming visual information early in the processing stream. Although integration of verbal and visual information is needed in all four conditions, it was specifically the condition in which both sources of information implied high risk that elicited a distinct positive shift in the ERP waveform, suggesting a tagging of the highest risk condition. This is compatible with the notion that the role of intuitive processes in risk perception is to implement a monitoring and alarm system (Schmälzle et al., 2017; Slovic et al., 2004). Consistent with this notion, fMRI studies (Häcker et al., 2014) revealed increased activation in the saliency network (Medford \& Critchley, 2010; Menon \& Uddin, 2010), and ERP studies point to early, affect-based, and incidental judgments (Schmälzle et al., 2012; Schmälzle et al., 2011). The picture that emerges is one in which the hypothesized risk perception system detects sources of risk and triggers an alarm signal, which in turn can regulate attention processes and generate feelings of risk reflected in explicit judgments (Loewenstein, Weber, Hsee, \& Welch, 2001; Slovic et al., 2004).

The notion of stereotype consistency provides a different perspective on the integration of risk information across multiple sources. The profile information about biographical, behavioral, and other risk-relevant data was selected to correspond to the low and high risk stereotype identified in previous research using verbal methods (Renner \& Schwarzer, 2003). The activation of stereotypes creates expectations about visual appearance (Hansen et al., 2017; Kunda \& Sherman-Williams, 1993; Kunda \& Thagard, 1996; Littlejohn \& Foss, 2009), which then match or clash with fast impressions driven by visual appearance (Willis \& Todorov, 2006). Thus, it seems possible that the consistency of verbal and visual risk information would predict ERP response patterns. However, although the differentiation between low and high visual risk following high verbal risk information is compatible with the stereotype consistency hypothesis, the lack of a differentiation for visual risk (low vs. high) in the low verbal risk conditions does not support this hypothesis. Thus, an explanation based on an expectancy violation account would require auxiliary assumptions - for instance, a negativity bias (i.e., stronger weighting of negative risk information; Cacioppo, Cacioppo, \& Gollan, 2014; Rozin \& Royzman, 2001). Overall, although future research needs to determine the integration of risk information across a broader array of experimental manipulations, the current pattern of findings is in favor of the risk sensitivity hypothesis.

The high temporal resolution of EEG is ideal for studying visual impressions, but the limited spatial resolution demands further research using fMRI to reveal the neural structures implicated in the integration of verbal-descriptive and visual information. In particular, more work is needed to substantiate the notion that the anterior temporal lobe is involved in integrating verbal and visual information, and to detail its 
relationship to structures of the salience network, that is, the amygdalae, anterior cingulate, and insular cortices. Other methodological modifications, such as presenting the visual information first and then analyzing neural responses to subsequent verbal statements, or using process-tracing methods to uncover sequential information sampling strategies, would help to provide a deeper understanding of how people "screen" potential partners. Such an approach would also help to further the understanding of different effects observed for ERPs and HIV risk ratings. Compared with ERP findings, self-report data were particularly sensitive to verbal risk information and did not show an interaction of visual and verbal risk. This may reflect, in part, the experimental design - that is, ERPs and self-report were measured at different times, or indicate different functions and sensitivities of physiological and self-report data (cf. Lang, 1968). Importantly, neither the current findings nor previous ones make a claim about the validity of the HIV risk stereotypes and visual impressions. Rather, reliance on feelings of risk has been dubbed as illusory control strategy providing ineffective protection against sexually transmitted infections. To our knowledge, only one study directly examined the ability to detect HIV positive individuals by presenting pictures and short vignettes from HIVpositive and HIV-negative people (Thompson, Kyle, Swan, Thomas, \& Vrungos, 2002). Participants were, on average, at chance in detecting HIV risk. Interestingly, receiving corrective feedback in this task as part of a broader intervention design was associated with increased condom use in a 3month follow-up. Thus, with the increasing number of online dating users, simulation online platforms may provide novel means for an experiential mode of experience, making visible the shortcomings of the strategy to gauge the riskiness or safety of potential partners on unreliable and superficial cues. Furthermore, including neural measures as well as ecological momentary assessment would allow insight on the processes underlying these judgements and to follow the dynamic of risk perception and behavior in everyday life.

\section{Summary}

Public health experts warn that online dating could escalate the spread of STIs because it is used by millions and creates conditions under which intuitive impressions are likely to emerge. Specifically, if people rely on intuitive impressions about a potential partner's risk as opposed to effective protection strategies, they are at risk for contracting HIV or STIs more broadly. In the present study, we took a novel approach to shed light on the processes involved in HIV risk perception online. We find that explicit verbal risk information altered brain processes of person perception specific to the condition signaling highest risk (i.e., high verbal and visual risk) relatively early in the processing stream $(\sim 270 \mathrm{~ms})$. These findings demonstrate how implicit processes associated with riskiness based on visual appearance can be contextualized and shaped by explicit biographical information about a person. Overall, simulating online dating platforms creates an experimental petri dish to study how multiple sources of biographical information are integrated and lead to intuitive impressions of risk.

Acknowledgements We thank Elena Heber for help with data collection and Alexander Barth, Frank Häcker, Tobias Flaisch, Ursula Kirmse, and Christoph Becker for valuable discussions.

Funding statement

This research was supported by the German Research Foundation (DFG, FOR 2374, http://gepris.dfg.de/gepris/projekt/273711585) granted to Britta Renner and Harald Schupp.

Open practices statement: The data for verbal profiles and materials are available at github.com/nomcomm/RiskProfiles_CABN. Additional files are available from the first author upon request. The images used cannot be shared due to copyright reasons.

\section{Appendix}

\section{EGI sensors' numbers comprising significant cluster effects}

Main effect of verbal risk. EGI sensor numbers comprising the occipitoparietal cluster: $45,53,60,78,79,80,81,86,87,88$, $89,90,96,97,98,99,100,101,104,105,106,107,108,109$, $110,111,112,113,114,115,116,117,118,119,124,125$, $126,127,128,129,130,131,132,138,139,143,144,186$, 257.

Main effect of visual risk. EGI sensor numbers comprising the occipital cluster: $123,124,125,134,135,136,137,138$, $145,146,147,148,149,150,151,156,157,158,159,160$, $161,165,166,167,168,169,170,171,174,175,176,177$, $178,179,187,188,189,190,191,199,200,201,208$

Main effect of visual risk. EGI sensor numbers comprising the central cluster: 8, 9, 16, 17, 24, 30, 41, 42, 43, 44, 45, 49, $51,52,53,58,59,60,65,66,72,78,79,80,81,131,132,142$, $143,144,154,155,163,164,173,181,182,183,184,185$, 186, 193, 194, 195, 196, 197, 198, 203, 204, 205, 206, 207, 257

Visual $\times$ Verbal Risk interaction effect. EGI sensor numbers comprising the anterior temporal cluster: $210,217,218$, $219,220,225,226,227,228,230,231,232,233,234,235$, $236,237,238,239,240$

\section{References}

Abdel Rahman, R. (2011). Facing good and evil: Early brain signatures of affective biographical knowledge in face recognition. Emotion, 11(6), 1397-1405.

Adams, R. B., Ambady, N., Nakayama, K., \& Shimojo, S. (2010). The science of social vision. Oxford, UK: Oxford University Press. 
Adolphs, R. (2003). Cognitive neuroscience of human social behaviour. Nature Reviews Neuroscience, 4(3), 165-178.

Adolphs, R. (2009). The social brain: Neural basis of social knowledge. Annual Review of Psychology, 60, 693-716.

Agocha, V. B., \& Cooper, M. L. (1999). Risk perceptions and safer-sex intentions: Does a partner's physical attractiveness undermine the use of risk-relevant information? Personality and Social Psychology Bulletin, 25(6), 751-765.

Allison, T., Puce, A., Spencer, D. D., \& McCarthy, G. (1999). Electrophysiological studies of human face perception. I: Potentials generated in occipitotemporal cortex by face and nonface stimuli. Cerebral Cortex, 9(5), 415-430.

Ambady, N., \& Rosenthal, R. (1992). Thin slices of expressive behavior as predictors of interpersonal consequences: A meta-analysis. Psychological Bulletin, 111(2), 256.

Barbeau, E. J., Taylor, M. J., Regis, J., Marquis, P., Chauvel, P., \& Liégeois-Chauvel, C. (2008). Spatio temporal dynamics of face recognition. Cerebral Cortex, 18(5), 997-1009.

Barth, A., Schmälzle, R., Renner, B., \& Schupp, H. T. (2013). Neural correlates of risk perception: HIV vs. leukemia. Frontiers in Behavioral Neuroscience, 7, 166.

Baum, J., Rabovsky, M., Rose, S. B., \& Abdel Rahman, R. (2018). Clear judgments based on unclear evidence: Person evaluation is strongly influenced by untrustworthy gossip. Emotion https://doi.org/10. 1037/emo0000545

Bellew, C. C., \& Todorov, A. (2007). Predicting political elections from rapid and unreflective face judgements. Proceedings of the National Academy of Sciences of the United States of America, 104, 1794817953.

Brooks, A. W., Huang, L., Kearney, S. W., \& Murray, F. E. (2014). Investors prefer entrepreneurial ventures pitched by attractive men. Proceedings of the National Academy of Sciences of the United States of America, 111(12), 4427-4431.

Bruch, E., Feinberg, F., \& Lee, K. Y. (2016). Extracting multistage screening rules from online dating activity data. Proceedings of the National Academy of Sciences of the United States of America, 113(38), 10530-10535.

Buhi, E. R., Klinkenberger, N., McFarlane, M., Kachur, R., Daley, E. M., Baldwin, J., . . . Rietmeijer, C. (2013). Evaluating the Internet as a sexually transmitted disease risk environment for teens: Findings from the communication, health, and teens study. Sexually Transmitted Diseases, 40(7), 528-533.

Cabecinha, M., Mercer, C. H., Gravningen, K., Aicken, C., Jones, K. G., Tanton, C., . . . Field, N. (2017). Finding sexual partners online: Prevalence and associations with sexual behaviour, STI diagnoses and other sexual health outcomes in the British population. Sexually Transmitted Infections, 93(8), 572-582.

Cacioppo, J. T., Cacioppo, S., \& Gollan, J. K. (2014). The negativity bias: Conceptualization, quantification, and individual differences. The Behavioral and Brain Sciences, 37(3), 309-310.

Calder, A., Rhodes, G., Johnson, M., \& Haxby, J. (2011). Oxford handbook of face perception. Oxford, UK: Oxford University Press.

Clark, J. (2015). Mobile dating apps could be driving HIV epidemic among adolescents in Asia Pacific, report says. BMJ, 351, h6493.

Collins, J. A., Koski, J. E., \& Olson, I. R. (2016). More than meets the eye: The merging of perceptual and conceptual knowledge in the anterior temporal face area. Frontiers in Human Neuroscience, 10, 189.

Collins, J. A., \& Olson, I. R. (2014a). Beyond the FFA: The role of the ventral anterior temporal lobes in face processing. Neuropsychologia, 61, 65-79.

Collins, J. A., \& Olson, I. R. (2014b). Knowledge is power: How conceptual knowledge transforms visual cognition. Psychonomic Bulletin \& Review, 21(4), 843-860.
Couch, D., \& Liamputtong, P. (2007). Online dating and mating: Perceptions of risk and health among online users. Health, Risk \& Society, 9(3), 275-294.

Couch, D., Liamputtong, P., \& Pitts, M. (2012). What are the real and perceived risks and dangers of online dating? Perspectives from online daters. Health, Risk \& Society, 14(7/8), 697-714.

Delorme, A., Rousselet, G. A., Macé, M. J.-M., \& Fabre-Thorpe, M. (2004). Interaction of top-down and bottom-up processing in the fast visual analysis of natural scenes. Brain Research. Cognitive Brain Research, 19(2), 103-113.

Eberhardt, J. L., Davies, P. G., Purdie-Vaughns, V. J., \& Johnson, S. L. (2006). Looking deathworthy: Perceived stereotypicality of Black defendants predicts capital-sentencing outcomes. Psychological Science, 17(5), 383-386.

Eleftheriou, A., Bullock, S., Graham, C. A., Stone, N., \& Ingham, R. (2016). Does attractiveness influence condom use intentions in heterosexual men? An experimental study. BMJ Open, 6(6), e010883.

Finkel, E. J., Eastwick, P. W., Karney, B. R., Reis, H. T., \& Sprecher, S. (2012). Online dating: A critical analysis from the perspective of psychological science. Psychological Science in the Public Interest: A Journal of the American Psychological Society, 13(1), $3-66$.

Fishbein, M., Hennessy, M., Yzer, M., \& Curtis, B. (2004). Romance and risk: Romantic attraction and health risks in the process of relationship formation. Psychology, Health \& Medicine, 9(3), 273-285.

Gilbert, D. T. (1998). Ordinary personology. In D. Gilbert, S. Fiske, \& G. Lindzey (Eds.), The handbook of social psychology (4th ed., Vol. 2, pp. 89-150). New York, NY: Random House.

Gold, R. S., Karmiloff-Smith, A., Skinner, M. J., \& Morton, J. (1992). Situational factors and thought processes associated with unprotected intercourse in heterosexual students. AIDS Care, 4(3), 305-323.

Greenwood, B. N., \& Agarwal, R. (2016). Matching platforms and HIV incidence: An empirical investigation of race, gender, and socioeconomic status. Management Science, 62(8), 2281-2303.

Häcker, F., Schmälzle, R., Renner, B., \& Schupp, H. T. (2014). Neural correlates of HIV risk feelings. Social Cognitive and Affective Neuroscience, 1-6. https://doi.org/10.1093/scan/nsu093(nsu093)

Hansen, K., Steffens, M. C., Rakic, T., \& Wiese, H. (2017). When appearance does not match accent: Neural correlates of ethnicityrelated expectancy violations. Social Cognitive and Affective Neuroscience, 12(3), 507-515.

Heijman, T., Stolte, I., Geskus, R., Matser, A., Davidovich, U., Xiridou, M., \& Schim van der Loeff, M. (2016). Does online dating lead to higher sexual risk behaviour? A cross-sectional study among MSM in Amsterdam, the Netherlands. BMC Infectious Diseases, 16, 288.

Heino, R. D., Ellison, N. B., \& Gibbs, J. L. (2010). Relationshopping: Investigating the market metaphor in online dating. Journal of Social and Personal Relationships, 27(4), 427-447.

Hennessy, M., Fishbein, M., Curtis, B., \& Barrett, D. W. (2007). Evaluating the risk and attractiveness of romantic partners when confronted with contradictory cues. AIDS and Behavior, 11(3), 479-490.

Ille, N., Berg, P., \& Scherg, M. (2002). Artifact correction of the ongoing EEG using spatial filters based on artifact and brain signal topographies. Journal of Clinical Neurophysiology: Official Publication of the American Electroencephalographic Society, 19(2), 113-124.

Junghöfer, M., Elbert, T., Tucker, D. M., \& Rockstroh, B. (2000). Statistical control of artifacts in dense array EEG/MEG studies. Psychophysiology, 37(4), 523-532.

Keller, M. L. (1993). Why don't young adults protect themselves against sexual transmission of HIV? Possible answers to a complex question. AIDS Education and Prevention: Official Publication of the International Society for AIDS Education, 5(3), 220-233.

Klepinger, D. H., Billy, J. O., Tanfer, K., \& Grady, W. R. (1993). Perceptions of AIDS risk and severity and their association with 
risk-related behavior among U.S. men. Family Planning Perspectives, 25(2), 74-82.

Kunda, Z., \& Sherman-Williams, B. (1993). Stereotypes and the construal of individuating information. Personality and Social Psychology Bulletin, 19(1), 90-99.

Kunda, Z., \& Thagard, P. (1996). Forming impressions from stereotypes, traits, and behaviors: A parallel-constraint-satisfaction theory. Psychological Review, 103(2), 284.

Lambon Ralph, M. A., Jefferies, E., Patterson, K., \& Rogers, T. T. (2016). The neural and computational bases of semantic cognition. Nature Reviews. Neuroscience. https://doi.org/10.1038/nrn.2016.150

Lang, P. J. (1968). Fear reduction and fear behavior: Problems in treating a construct. In J. M. Shlien (Ed.), Research in psychotherapy (pp. 90-102). Washington, DC: American Psychological Association.

Lin, K.-H., \& Lundquist, J. (2013). Mate selection in cyberspace: The intersection of race, gender, and education. The American Journal of Sociology, 119(1), 183-215.

Linville, P. W., \& Carlston, D. E. (1994). Social cognition of the self. In P. G. Devine, D. L. Hamilton, \& T. M. Ostrom (Eds.), Social cognition: Impact on social psychology (pp. 143-193). San Diego, CA: Academic Press.

Littlejohn, S., \& Foss, K. (Eds.). (2009). Encyclopedia of communication theory. Thousand Oaks, CA: SAGE Publications.

Loewenstein, G., Weber, E. U., Hsee, C. K., \& Welch, N. (2001). Risk as feelings. Psychological Bulletin, 127(2), 267-286.

Luo, Q.L., Wang, H.L., Dzhelyova, M., Huang, P., \& Mo, L. (2016). Effect of affective personality information on face processing: Evidence from ERPs. Frontiers in Psychology, 7, 810.

Maris, E., \& Oostenveld, R. (2007). Nonparametric statistical testing of EEG- and MEG-data. Journal of Neuroscience Methods, 164(1), 177-190.

Maticka-Tyndale, E. (1991). Sexual scripts and AIDS prevention: Variations in adherence to safer-sex guidelines by heterosexual adolescents. Journal of Sex Research, 28(1), 45-66.

McFarlane, M., Bull, S. S., \& Rietmeijer, C. A. (2000). The Internet as a newly emerging risk environment for sexually transmitted diseases. JAMA: The Journal of the American Medical Association, 284(4), 443-446.

Medford, N., \& Critchley, H. D. (2010). Conjoint activity of anterior insular and anterior cingulate cortex: Awareness and response. Brain Structure \& Function, 214(5/6), 535-549.

Menon, V., \& Uddin, L. Q. (2010). Saliency, switching, attention and control: A network model of insula function. Brain Structure \& Function, 214(5/6), 655-667.

Naumann, L. P., Vazire, S., Rentfrow, P. J., \& Gosling, S. D. (2009). Personality judgments based on physical appearance. Personality and Social Psychology Bulletin, 35(12), 1661-1671.

Olson, I. R., \& Marshuetz, C. (2005). Facial attractiveness is appraised in a glance. Emotion, 5(4), 498-502.

Olson, I. R., McCoy, D., Klobusicky, E., \& Ross, L. A. (2013). Social cognition and the anterior temporal lobes: A review and theoretical framework. Social Cognitive and Affective Neuroscience, 8(2), 123133.

Ortiz-Martínez, Y., Buelvas-Pérez, A., Martínez-Torres, A., VásquezRada, K., \& Carrascal-Angelo, A. E. (2018). Dating apps and increased sexual risk behaviors while traveling: Challenges and opportunities for public health. Travel Medicine and Infectious Disease, 24, 7 .

Osterhout, L., Bersick, M., \& McLaughlin, J. (1997). Brain potentials reflect violations of gender stereotypes. Memory \& Cognition, 25(3), 273-285.

Peyk, P., De Cesarei, A., \& Junghöfer, M. (2011). ElectroMagnetoEncephalography software: Overview and integration with other EEG/MEG toolboxes. Computational Intelligence and Neuroscience, 2011, 861705.
Prestage, G., Zablotska, I., Bavinton, B., Grulich, A., Keen, P., Murphy, D., ... Guy, R. (2016). Previous and future use of HIV self-testing: a survey of Australian gay and bisexual men. Sexual Health, 13(1), $55-62$.

Renner, B., Gamp, M., Schmälzle, R., \& Schupp, H. T. (2015). Health risk perception. International Encyclopedia of the Social \& Behavioral Sciences, 10, 702-709.

Renner, B., Schmälzle, R., \& Schupp, H. T. (2012). First impressions of HIV risk: It takes only milliseconds to scan a stranger. PLOS ONE, 7(1), e30460.

Renner, B., \& Schwarzer, R. (2003). Risikostereotype, Risikowahrnehmung und Risikoverhalten im Zusammenhang mit HIV [Risk stereotypes, risk perception, and risk behavior in the context of HIV]. Zeitschrift Für Gesundheitspsychologie, 11(3), $112-121$.

Rosenfeld, M. J., \& Thomas, R. J. (2012). Searching for a mate: The rise of the Internet as a social intermediary. American Sociological Review, 77(4), 523-547.

Rozin, P., \& Royzman, E. B. (2001). Negativity bias, negativity dominance, and contagion. Personality and Social Psychology Review: An Official Journal of the Society for Personality and Social Psychology, Inc, 5(4), 296-320.

Schmälzle, R., Hartung, F., Barth, A., Imhof, M. A., Kenter, A., Renner, B., \& Schupp, H. T.. (2019). Visual cues that predict intuitive risk perception in the case of HIV. PLoS ONE, 14(2), e0211770.

Schmälzle, R., Renner, B., \& Schupp, H. T. (2012). Neural correlates of perceived risk: The case of HIV. Social Cognitive and Affective Neuroscience, 7(6), 667-676.

Schmälzle, R., Renner, B., \& Schupp, H. T. (2017). Health risk perception and risk communication. Policy Insights From the Behavioral and Brain Sciences. https://doi.org/10.1177/2372732217720223

Schmälzle, R., Schupp, H. T., Barth, A., \& Renner, B. (2011). Implicit and explicit processes in risk perception: Neural antecedents of perceived HIV risk. Frontiers in Human Neuroscience, 5(1), 1.

Schupp, H. T., Flaisch, T., Stockburger, J., \& Junghöfer, M. (2006). Emotion and attention: Event-related brain potential studies. Progress in Brain Research, 156, 31-51.

Siegel, K., Lekas, H.-M., Onaga, M., Verni, R., \& Gunn, H. (2017). The strategies of heterosexuals from large metropolitan areas for assessing the risks of exposure to HIV or other sexually transmitted infections from partners met online. AIDS Patient Care and STDs, 31(4), 182-195.

Slovic, P., Finucane, M. L., Peters, E., \& MacGregor, D. G. (2004). Risk as analysis and risk as feelings: Some thoughts about affect, reason, risk, and rationality. Risk Analysis: An Official Publication of the Society for Risk Analysis, 24(2), 311-322.

Stokes, D. E. (2011). Pasteur's quadrant: Basic science and technological innovation. Washington, DC: Brookings Institution Press.

Suess, F., Rabovsky, M., \& Rahman, R. A. (2015). Perceiving emotions in neutral faces: Expression processing is biased by affective person knowledge. Social Cognitive and Affective Neuroscience, 10, 531536.

Thompson, S. C., Kyle, D., Swan, J., Thomas, C., \& Vrungos, S. (2002). Increasing condom use by undermining perceived invulnerability to HIV. AIDS Education and Prevention: Official Publication of the International Society for AIDS Education, 14(6), 505-514.

Todorov, A. (2017). Face value: The irresistible influence of first impressions. Princeton, NJ: Princeton University Press.

Toma, C. L., Hancock, J. T., \& Ellison, N. B. (2008). Separating fact from fiction: An examination of deceptive self-presentation in online dating profiles. Personality and Social Psychology Bulletin, 34(8), 1023-1036.

Trautner, P., Dietl, T., Staedtgen, M., Mecklinger, A., Grunwald, T., Elger, C. E., \& Kurthen, M. (2004). Recognition of famous faces in the medial temporal lobe: An invasive ERP study. Neurology, 63(7), $1203-1208$. 
Tsuchiya, N., Kawasaki, H., Oya, H., Howard, M. A., III, \& Adolphs, R. (2008). Decoding face information in time, frequency and space from direct intracranial recordings of the human brain. PLOS ONE, 3(12), e3892.

Uleman, J. S., Blader, S. L., \& Todorov, A. (2006a). Implicit impressions. In R. R. Hassin, J. S. Uleman, \& J. A. Bargh (Eds.), The new unconscious (pp. 362-392). Oxford, UK: Oxford University Press.

Uleman, J. S., Blader, S. L., \& Todorov, A. (2006b). Implicit impressions. In The New Unconscious. Oxford University Press.

UNAIDS. (2016). http://paperpile.com/b/SJQFfM/vULX. Prevention gap report. Retrieved from http:/www.unaids.org/en/resources/ documents/2016/prevention-gap.

Wang, Y., Collins, J. A., Koski, J., Nugiel, T., Metoki, A., \& Olson, I. R. (2017). Dynamic neural architecture for social knowledge retrieval. Proceedings of the National Academy of Sciences of the United States of America, 114(16), E3305-E3314.
White, K. R., Crites, S. L., Jr, Taylor, J. H., \& Corral, G. (2009). Wait, what? Assessing stereotype incongruities using the N400 ERP component. Social Cognitive and Affective Neuroscience, 4(2), 191198.

Willis, J., \& Todorov, A. (2006). First impressions: Making up your mind after a 100-ms exposure to a face. Psychological Science, 17(7), $592-598$.

Publisher's note Springer Nature remains neutral with regard to jurisdictional claims in published maps and institutional affiliations. 